\title{
Um estudo sobre a Eficiência da Quadratura Gaussiana na Integração Singular com o Método dos Elementos de Contorno
}

\author{
Barbosa, J. P. ${ }^{1,2}$; Loeffler, C. F. ${ }^{1^{\star}}$; Bulcão, A, ${ }^{3}$ \\ 1 Programa de Pós-Graduação em Engenharia Mecânica, Universidade Federal do Espírito Santo, Vitória, ES, Brasil. \\ 2 Instituto Federal do Espírito Santo - IFES Campus São Mateus, ES, Brasil. \\ 3 PETROBRAS, CENPES - Centro de Pesquisa e Desenvolvimento L. A. M. de Mello, Rio de Janeiro, RJ, Brasil. \\ *e-mail: carlosloeffler@bol.com.br
}

\begin{abstract}
Resumo
Neste trabalho, apesar da existência de diversas técnicas voltadas para a solução numérica de integrais singulares, objetiva-se testar a eficiência da quadratura de Gauss na solução da integral de linha imprópria que surge nos procedimentos de integração típicos do Método dos Elementos de Contorno, quando se realiza a integração da solução fundamental ao longo de um elemento que contem seu próprio ponto de origem, denominado ponto fonte. Nestes testes é possível observar o quanto a presença de singularidades, mesmo fracas, impõem perturbação aos resultados, sobretudo no caso de elementos não retilíneos, como os que aqui são aqui utilizados.
\end{abstract}

\begin{abstract}
In this work, despite the existence of several techniques aimed at the numerical solution of singular integrals, we aim to test the Gauss quadrature efficiency in the solution of the improper line integral that arises in the integration procedures typical of the Contour Element Method, when The fundamental solution is integrated along an element that contains its own point of origin, called the source point. In these tests it is possible to observe how much the presence of singularities, even weak ones, impose disturbance to the results, especially in the case of non-rectilinear elements, such as those that are approached here.
\end{abstract}

Keywords (Palavras chaves): Elementos de Contorno, Quadratura de Gauss, Elementos Quadráticos.

\section{Introdução}

A integração numérica é um dos tópicos de maior relevância no contexto do Método dos Elementos de Contorno (MEC) [1]. Em princípio, já que o método reduz o problema a ser resolvido em uma dimensão, seria de se esperar que as integrações fossem mais simples. Contudo, embora seja a razão da precisão elevada do método, a solução fundamental também é causa de problemas relacionados à sua integração ao longo do elemento de contorno, pois possui a característica peculiar de ser singular. Assim, as integrais do MEC realizadas ao longo de um elemento que contém o ponto de origem da solução fundamental, dito ponto fonte, são integrais impróprias, embora convergentes. Não bastasse isso, a solução fundamental torna-se complicada e de difícil trato, quanto mais complexo for o problema a ser resolvido.
É possível fazer o cálculo analítico das integrais do MEC em certos casos particulares; contudo, torna-se praticamente inviável realizá-lo para elementos de ordem superior, isto é, onde a variação da variável básica ao longo do elemento não é considerada constante. Um agravante surge no caso de elementos com geometria não retilínea, como no caso de elementos isoparamétricos quadráticos ou superiores em que a função que realiza a transformação das coordenadas globais num sistema estratégico local, o Jacobiano, não é mais um valor constante e sim uma função da forma geométrica do elemento.

Tais dificuldades foram motivação para diversos trabalhos de pesquisa e pode-se afirmar que esses foram resolvidos satisfatoriamente e de diversas formas. As mais usuais são a partição de domínio, o esquema de integração de Telles e a simples adoção 
da coordenada do ponto fonte fora do elemento de contorno.

Neste trabalho são testados elementos quadráticos isoparamétricos. Ressalta-se que apesar da existência de todas essas técnicas citadas, objetiva-se testar a eficiência da quadratura de Gauss na solução de uma integral de linha imprópria. Observar-se-á o quanto a presença de singularidades, mesmo fracas, impõe oscilações nos resultados numéricos, particularmente quando são usados elementos de ordem superior, como os elementos quadráticos aqui empregados.

\section{Método dos Elementos de Contorno}

O computador permitiu a acessibilidade de processamento e solução de sistemas algébricos de grande porte, através do desenvolvimento de métodos matemáticos aproximados, especialmente àqueles ligados à ideia de discretização do domínio continuo. Discretizar significa substituí-lo por um conjunto finito de pontos, que passam assim a representá-lo. Este processo, evidentemente, deve ser feito de forma matematicamente consistente, de modo que possa se garantir erros desprezíveis nesta transformação. Em outras palavras, o modelo numérico resultante da discretização deve refletir, com boa aproximação, o modelo matemático original. Também com o aumento do número de pontos de discretização deve haver uma convergência dos resultados do modelo aproximado para uma normalmente indisponível solução exata.

Dentre as técnicas numéricas mais utilizadas, uma delas se destaca por uma série de características vantajosas em que se destaca a redução de uma dimensão na representação do problema: o Método dos Elementos de Contorno [2, 3].

Utilizando um procedimento matemático consistente, baseado em princípios da Teoria das Equações Integrais, o MEC transforma a equação diferencial associada ao modelo matemático em uma expressão integral, onde os limites da integração encontram-se somente sobre o contorno físico do problema.

No caso de problemas bidimensionais, por exemplo, o MEC discretiza apenas o contorno do domínio físico, usando segmentos de linha, que podem ser retilíneos ou curvilíneos. Estes segmentos são os denominados elementos de contorno. Ao longo destes elementos se situam os nós geométricos, responsáveis pela localização espacial do elemento com relação a um sistema de coordenadas, e também os nós funcionais, que caracterizam o comportamento da variável física de modo aproximado. A figura 1 ilustra um tipo linear de elemento de contorno, em que o nó funcional é central e os nos geométricos se encontram nas extremidades.

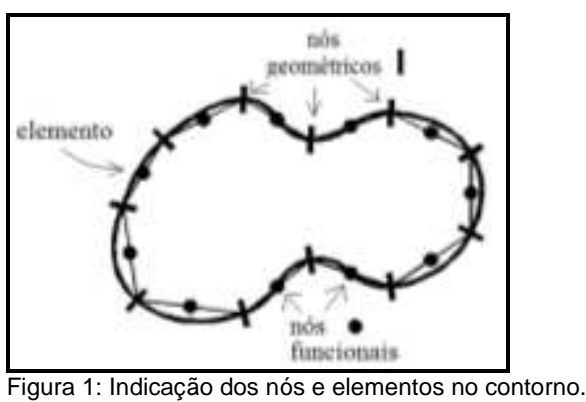

O MEC apresenta vantagens importantes para solução de diversas aplicações na engenharia e na física matemática. Além de melhor captar concentração de esforços, ajusta-se de modo ímpar a solução de problemas com regiões infinitas e semi-infinitas e casos em que se precisa movimentar a fronteira de alguma forma, como ocorre nas simulações do contato entre partes e no acompanhamento da formação de trincas.

O MEC também apresenta uma precisão elevada na maior parte dos problemas em que se aplica com naturalidade. Isto porque o método perde atratividade nos casos em que o domínio é relativamente pequeno face ao contorno circundante, como ocorre em problemas delgados. Certos modelos matemáticos que não possuem operadores auto adjuntos também não se mostram adequados a aplicação do MEC.

Já a razão para sua alta precisão pode ser compreendida à luz do Método dos Resíduos Ponderados. Este método permite a dedução das principais técnicas numéricas discretas através de um princípio único, que arrola a minimização de resíduos através de funções auxiliares. No MEC, a função de ponderação é a solução fundamental, que é a solução de um problema correlato, ou seja, um problema similar ao que se deseja resolver, conferindo efetividade na obediência ao princípio de minimização residual.

\section{Equação Integral para a Equação de Laplace}

A equação de Laplace é um caso particular, mas extremamente importante da Equação de Campo Escalar Generalizada [2, 4], pois modela diversos problemas pertinentes à mecânica dos sólidos, à mecânica dos fluidos, à termociência e à eletricidade.

Assim, para formulá-la considere um domínio bidimensional $\Omega(\mathrm{X})$ que represente um campo térmico ou mecânico no estado estacionário, que possui propriedades homogêneas e isotrópicas e não há fontes, coletores ou ações de domínio externo que contribuam diretamente para o campo [5]. Tomando a variável básica $\mathrm{u}(\mathrm{X})$ como um potencial escalar, a equação diferencial associada a este problema é chamada de Equação de Laplace. 
Existem duas formas de se deduzir a equação integral inversa típica do método dos elementos de contorno em sua forma direta, a mais difundida: através dos princípios da Teoria das Equações integrais ou através de uma sentença de resíduos ponderados. Seja qual for a tática usada, chega-se a seguinte equação (1):

$$
c(\xi) u(\xi)+\int_{\Gamma} u(X) q^{*}(\xi ; X) d \Gamma=\int_{\Gamma} q(X) u^{*}(\xi ; X) d \Gamma
$$

O domínio $\Omega(X)$ é limitado por um contorno $\Gamma(X)$, onde condições de potencial são prescritas em $\Gamma_{\mathrm{u}}(\mathrm{X})$ e derivadas normais do potencial $\mathrm{q}(\mathrm{X})$ são aplicadas no contorno complementar $\Gamma_{\mathrm{q}}(\mathrm{X})$. A função auxiliar $\mathrm{u}^{*}(\xi ; X)$ é a solução fundamental e sua derivada normal é expressa por $\mathrm{q}^{*}(\xi ; X)$; o argumento dessas funções considera o ponto fonte $\xi$, que é um ponto arbitrário no domínio X [6], pois tais funções dependem da distância euclidiana entre dois pontos. O coeficiente $\mathrm{c}(\xi)$ depende da suavidade do contorno [2].

\section{Discretização do Contorno}

Com a discretização, realizada apenas no contorno, a equação (1) fica da seguinte forma:

$$
c(\xi) u(\xi)+\sum_{j=1}^{N E} \int_{\Gamma_{j}} u(X) q^{*}(\xi ; X) d \Gamma_{j}=\sum_{j=1}^{N E} \int_{\Gamma_{j}} q(X) u^{*}(\xi ; X) d \Gamma_{j}
$$

$N E$ assinala a quantidade de elementos de contorno que compõem a discretização. No caso dos campos de variáveis $\mathrm{u}(\mathrm{X})$ e $\mathrm{q}(\mathrm{X})$ serem aproximados por funções quadráticas e os elementos no contorno também - os elementos isoparamétricos - pode-se escrever a equação (2) em uma forma matricial apropriada:

$$
\begin{gathered}
c(\xi) u(\xi)+\sum_{e=1}^{N E}\left[\begin{array}{lll}
U_{1} & U_{2} & U_{3}
\end{array}\right] \int_{\Gamma_{e}}^{e}\left[\begin{array}{c}
N_{1} \\
N_{2} \\
N_{3}
\end{array}\right] q^{*}(\xi ; X) d \Gamma_{j}= \\
\sum_{e=1}^{N E}\left[\begin{array}{lll}
Q_{1} & Q_{2} & Q_{3}
\end{array}\right]_{\Gamma_{e}}^{e}\left[\begin{array}{c}
N_{1} \\
N_{2} \\
N_{3}
\end{array}\right] u^{*}(\xi ; X) d \Gamma_{j}
\end{gathered}
$$

Seja qual for o tipo de aproximação usada, a aplicação do MEC na Equação de Laplace resulta num sistema misto envolvendo potenciais $U$ nos nós, bem como derivadas normais ou fluxos $Q$ também nodais [2, 4, 5]. O número de incógnitas dos dois tipos é compensado pela prescrição de valores conhecidos em número suficiente para gerar um sistema composto por matrizes quadradas, na forma:

$$
[H]\{U\}=[G]\{Q\}
$$

Os coeficientes das matrizes $H$ e $G$ também são chamados de coeficientes de influência e a precisão do MEC está fortemente relacionada a obtenção correta de tais coeficientes.

\section{Interpolação nos Elementos}

É usual escrever-se a variação do campo e das variáveis do campo através de funções de interpolação ou funções de forma $\phi_{i}$, que são expressas em termos de uma variável adimensional $\eta(\mathrm{x})[2]$ :

$$
\begin{aligned}
& u_{e}(x)=U_{1}^{e} \phi_{1}+U_{2}^{e} \phi_{2}+U_{3}^{e} \phi_{3} \\
& =U_{1} \frac{1}{2}\left(\eta^{2}-\eta\right)+U_{2}\left(1-\eta^{2}\right)+U_{3} \frac{1}{2}\left(\eta^{2}+\eta\right) \\
& q_{e}(x)=Q_{1}^{e} \phi_{1}+Q_{2}^{e} \phi_{2}+Q_{3}^{e} \phi_{3} \\
& =Q_{1} \frac{1}{2}\left(\eta^{2}-\eta\right)+Q_{2}\left(1-\eta^{2}\right)+Q_{3} \frac{1}{2}\left(\eta^{2}+\eta\right)
\end{aligned}
$$

Assim a integração dos núcleos das integrais constantes na equação deve ser adaptada, pois as funções $\eta$ são adimensionais e normalizadas no intervalo $(-1,1)$. É necessário relacionar $\mathrm{d} \Gamma(\mathrm{X})$, representado no sistema de coordenadas globais $\mathrm{X}$, com tal sistema de coordenadas, através do Jacobiano $\mathrm{J}(\mathrm{X})$ da transformação de coordenadas, dado por:

$$
d \Gamma=|J| d \eta
$$

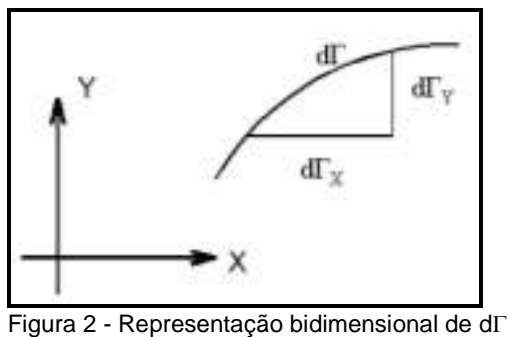

De acordo com os princípios da geometria diferencial e tomando a figura 2 como base, tem-se que $\mathrm{d} \Gamma(\mathrm{X})$ pode ser aproximado pela seguinte expressão:

$d \Gamma=\sqrt{\left(\frac{d \Gamma_{X}}{d \eta}\right)^{2}+\left(\frac{d \Gamma_{Y}}{d \eta}\right)^{2}} d \eta$

As derivadas do Jacobiano são dadas a seguir:

$$
\begin{aligned}
& \frac{\partial \Gamma_{x}}{\partial \eta}=\frac{\partial N_{1}}{\partial \eta} x_{1}+\frac{\partial N_{2}}{\partial \eta} x_{2}+\frac{\partial N_{3}}{\partial \eta} x_{3} \\
& \frac{\partial \Gamma_{y}}{\partial \eta}=\frac{\partial N_{1}}{\partial \eta} y_{1}+\frac{\partial N_{2}}{\partial \eta} y_{2}+\frac{\partial N_{3}}{\partial \eta} y_{3}
\end{aligned}
$$

Desenvolvendo-se as equações (9) e (10) e substituindo na equação (7) obtém-se o Jacobiano da transformação, que pode não ser um valor constante ao longo dos elementos de contorno:

$$
\begin{aligned}
& |J|=\left\{\left[\left(x_{1}-2 x_{2}+x_{3}\right) \eta+\frac{1}{2}\left(x_{3}-x_{1}\right)\right]^{2}+\right. \\
& \left.\left[\left(y_{1}-2 y_{2}+y_{3}\right) \eta+\frac{1}{2}\left(y_{3}-y_{1}\right)\right]^{2}\right\}^{1 / 2}
\end{aligned}
$$


Os coeficientes das matrizes típicas do MEC são compostos por:

$$
\begin{aligned}
& h_{j}^{k}=\int_{\Gamma_{j}} N_{k} q^{*}(\xi ; X) J_{j} d \eta \\
& g_{j}^{k}=\int_{\Gamma_{j}} N_{k} u^{*}(\xi ; X) J_{j} d \eta
\end{aligned}
$$

O núcleo dos coeficientes $h_{j}$ é constituído da derivada do raio vetor com a normal ao contorno.

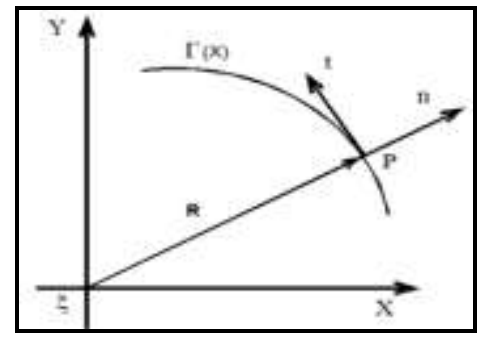

Figura 3 - Representação dos vetores tangente e normal a curva $\Gamma(\mathrm{X})$.

$\mathrm{Na}$ figura 3 apresentam-se os vetores tangente $\mathrm{t}(\mathrm{X}) \mathrm{e}$ normal $\mathrm{n}(\mathrm{X})$ à curva $\Gamma(\mathrm{X})$ num ponto campo $\mathrm{P}$. $\mathrm{Na}$ origem do sistema de coordenadas adotado encontrase $o$ ponto $\xi$, sendo $\mathbf{R}$ o vetor posição de $\xi$ ao ponto $\mathrm{P}$.

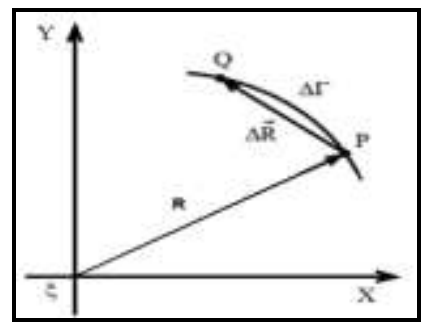

Figura 4 - Representação do vetor $\mathrm{PQ}$ na curva $\Gamma(\mathrm{X})$.

De acordo com a figura 4, pode-se expressar uma relação entre $\mathbf{R}$ e a curva $\Gamma(\mathrm{X})$, fazendo-se com que o ponto $Q$ se desloque em direção a $P$. Quando $Q \rightarrow P$, tem-se que $\Delta \Gamma(X) \rightarrow 0$. Desta forma a direção da corda $P Q$ tende para a direção da tangente à curva em $P$. Matematicamente:

$$
\frac{d \vec{R}}{d \Gamma}=\lim _{\Delta \Gamma \rightarrow 0} \frac{\Delta \vec{R}}{\Delta \Gamma}=t=\frac{d \vec{R}}{d \eta} \frac{d \eta}{d \Gamma}=\frac{d \vec{R}}{d \eta} \frac{1}{J}
$$

Para a determinação do vetor normal utiliza-se dos conceitos do produto escalar e do produto vetorial entre os vetores tangente e normal que resultam em:

$$
n=\frac{1}{J}\left[\frac{d Y}{d \eta} \vec{i}-\frac{d X}{d \eta} \vec{j}\right]
$$

\section{Integração Numérica}

Comumente, faz-se o cálculo numérico das integrais usando a Quadratura de Gauss quando o ponto fonte não se encontra no mesmo elemento em que se processa a integração. A integração analítica é reservada apenas para o cálculo dos coeficientes $G_{j}$. Isto porque os coeficientes $H_{j}$, cuja integral é mais fortemente singular, pode ser calculado pela soma dos coeficientes das linhas correspondentes, segundo o procedimento da obediência à imposição de um campo potencial constante [7], ou seja, leva-se em conta que quando um campo uniforme de potencial é aplicado ao longo de um domínio físico finito as derivadas do potencial devem ser nulas em todo o domínio físico:

$$
[H]\{U\}=\{0\}
$$

Dessa forma, tanto a integral mais fortemente singular $\mathrm{H}_{\mathrm{j}}$ quanto $\mathrm{o}$ valor do coeficiente $\mathrm{c}(\xi)$ podem ser calculados indiretamente. Já no caso da integral $G_{j}$, embora mais fracamente singular, seu cálculo para elementos quadráticos e de ordem superior é mais elaborado. A Quadratura de Gauss pode ser usada, mas, como será mostrado, há demanda de um número significativo de pontos de integração [3]. Tal esquema de integração possui a vantagem de calcular de forma precisa as integrais de funções polinomiais de graus $\mathrm{p}=2 \mathrm{NG}+1$. Em uma dimensão, como a que é aplicada no MEC em problemas bidimensionais é dada por:

$$
I=\int_{-1}^{1} f(x) d x=\sum_{p=1}^{N G} W_{p} f\left(\zeta_{p}\right)
$$

Onde NG é o número de pontos de integração, $\mathrm{W}_{\mathrm{p}}$ são os pesos da Quadratura de Gauss e $\xi_{p}$ são pontos de integração. Os pontos e os pesos são tabelados considerando um intervalo normalizado $(-1,1)$. Ressalta-se que o núcleo das integrais do MEC não é polinomial e que no caso da integral imprópria, é preciso calcular o que geometricamente representaria a área sobre a curva de modo correto, para evitar erros numéricos significativos.

\section{Exemplos}

\subsection{Exemplo 1}

Resolve-se inicialmente uma chapa engastada numa extremidade e sujeita a uma deformação constante aplicada na direção X, como mostra a figura 5. Os deslocamentos nesta direção variam linearmente. O problema é muito simples, mas afere a consistência do modelo de solução em que a integral fracamente singular é resolvida numericamente.

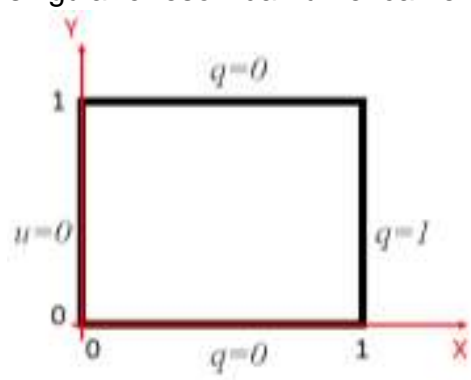

Figura 5 - Geometria e condições de contorno do exemplo 1. 
Com a aplicação de condições naturais nulas nas arestas horizontais, o problema fica unidimensional.

Discretizou-se o problema físico, foram empregadas três malhas diferentes com elementos quadráticos: a mais simples, com 20 elementos com 44 pontos nodais; uma malha intermediária com 40 elementos com 84 pontos nodais; e uma malha mais elaborada com 80 elementos e 164 pontos nodais.

Na figura 6 observa-se que o erro médio relativo para o cálculo do potencial ao longo da direção $x$ foi muito pequeno, embora maior do que se esperaria num caso muito simples como esse. Apenas com o aumento do número de pontos de Gauss a malha menos refinada pode obter erros bastante reduzidos.

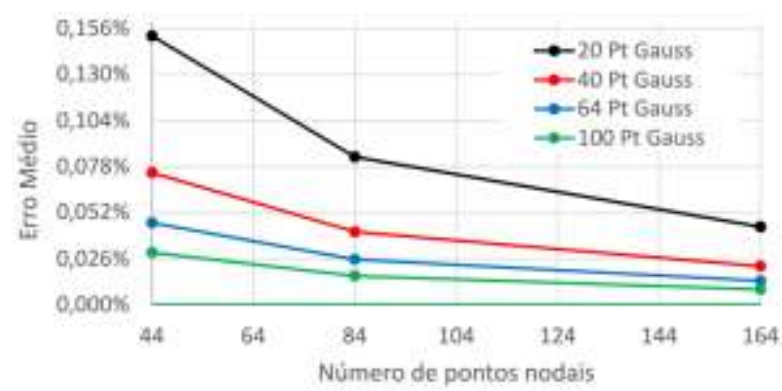

Figura 6 - Erro Médio do Potencial no exemplo 1.

Para a derivada do potencial na aresta vertical direita, que significaria a deformação no engaste, os erros são maiores conforme se observa na figura 7 .

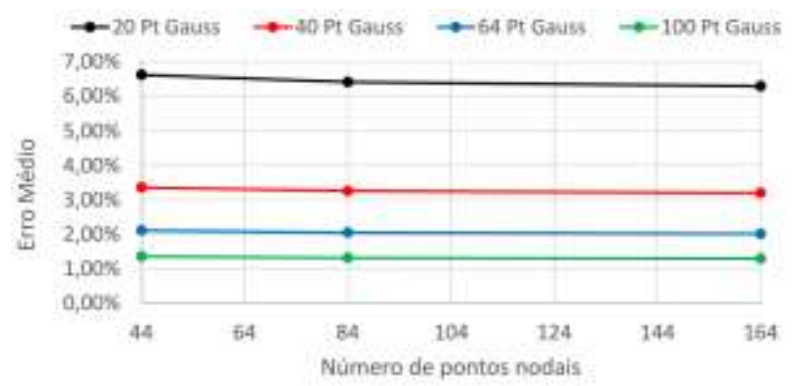

Figura 7 - Erro Médio da Derivada Potencial no exemplo 1.

O nível de erro elevado se deve as oscilações nos valores dos pontos nodais, observados na figura 8 .

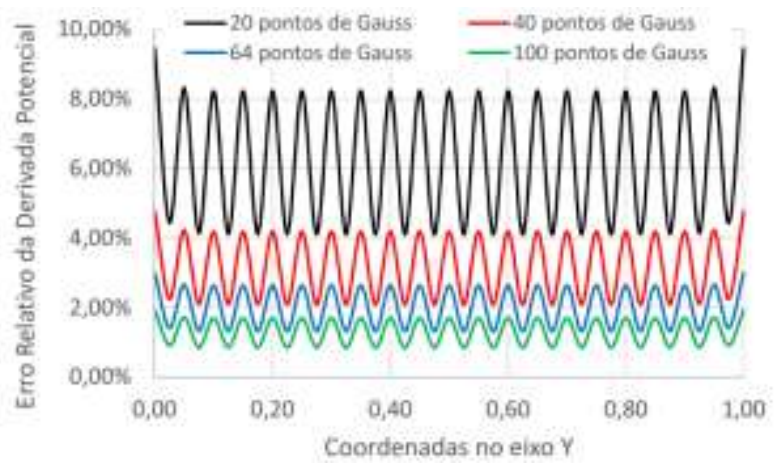

Figura 8 - Erro Relativo da Derivada Potencial do exemplo 1.
O refinamento da malha de contorno em nada afetou os resultados; daí conclui-se que o problema se deve certamente a questão da integração, pois o aumento dos pontos de Gauss melhorou a solução.

\subsection{Exemplo 2}

Nesta simulação uma chapa em forma semicircular, isolada em seu contorno curvo é submetido a um campo descontinuo de temperatura, conforme mostrado na figura 9.

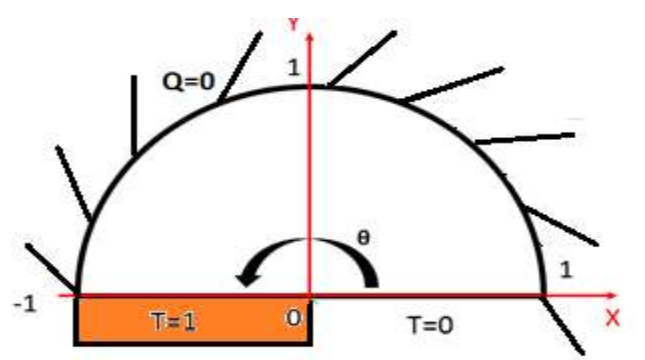

Figura 9 - Geometria e condições de contorno do exemplo 2.

Este problema possui solução analítica para temperatura e fluxos, dados por:

$T=\frac{1}{\pi} \theta \quad ; \quad q=-k \frac{\partial T}{\partial n}=\frac{k}{x} \frac{1}{\pi}$

Além da singularidade, a condição de isolamento no limite circular impõe um fluxo de calor independente do ângulo $\theta$, variando com uma magnitude inversamente proporcional ao raio. Sua solução é, portanto, uma função hiperbólica.

Para a discretização, três malhas distintas com elementos quadráticos foram usadas: a mais simples têm 25 elementos com 53 nós; a malha intermediária possui 50 elementos com 103 pontos nodais; a mais refinada tem 103 elementos e 209 pontos nodais.

Inicialmente são apresentados os resultados para o cálculo do potencial na aresta circular. A abscissa do gráfico contempla os pontos nodais enquanto a ordenada apresenta o erro relativo. Quatro simulações com diferentes quantidades de pontos de Gauss são apresentados. Observa-se que o nível de erro é bastante pequeno já para a malha mais pobre e se reduz monotonicamente tanto em função do aumento da quantidade de nós quanto pela introdução de maior número de pontos de Gauss conforme a figura 10.

Na figura 11 é possível identificar o comportamento da curva de erro da derivada do potencial na aresta retilínea. Neste caso, foram excluídos os nós duplos da descontinuidade do potencial e os dois nós imediatos, pois o valor cresce ao infinito e não é possível conferir quantitativamente a precisão do método. Usando-se este critério, e possível igualmente constatar que há convergência da solução numérica. 


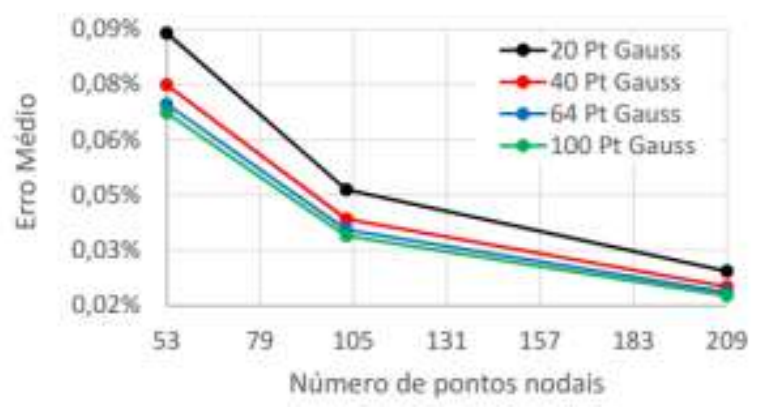

Figura 10 - Erro Médio do Potencial no exemplo 2.

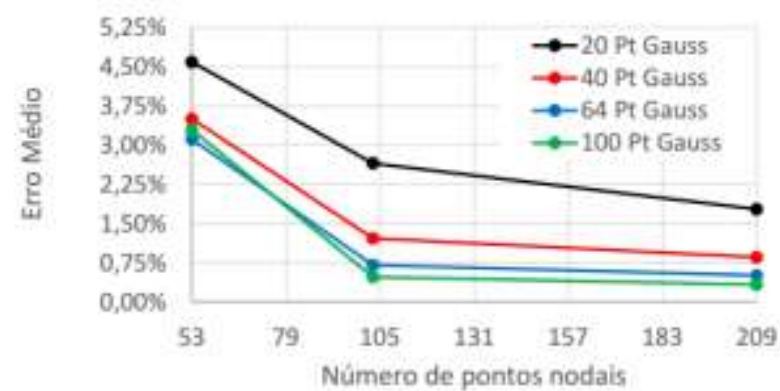

Figura 11 - Erro Médio da Derivada Potencial no exemplo 2.

Contudo, o valor do erro cresce no cálculo desta grandeza, tanto por força da ausência do esquema de integração eficaz, mais principalmente por conta da descontinuidade, que faz os valores oscilarem em regiões próximas. O refinamento não elimina esse problema, mas concentra esse efeito cada vez mais na proximidade da descontinuidade local conforme podese observar na figura 12. A eliminação deste problema usando adaptatividade e outras técnicas auxiliares foram propostas na literatura especializada [8].

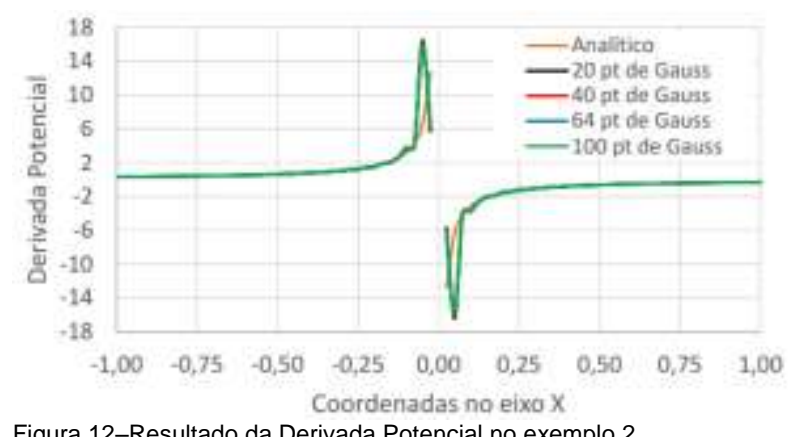

Figura 12-Resultado da Derivada Potencial no exemplo 2.

O comportamento da solução numérica para a derivada do potencial ao longo da aresta retilínea para a malha mais refinada é mostrada na figura 12 , considerando diversas quantidades de pontos de Gauss. Uma curva de erro local da derivada do potencial para esta mesma aresta usando a mesma malha pode ser visualizada na figura 13.

\section{Conclusões}

A presença de oscilações numéricas em modelos discretos de ordem mais elevada é um fenômeno observado em diversos métodos, incluindo o MEC. Pode-se aferir pelas experiências aqui apresentadas que a melhoria da integração numérica apresenta um controle relativo destas, embora não as elimine completamente. Contudo, em termos de precisão dos resultados, visando aplicações na Engenharia e na Física, o nível de erro apresentado é reduzido, indicando que mesmo a quadratura gaussiana simples é suficiente para gerar respostas satisfatórias.

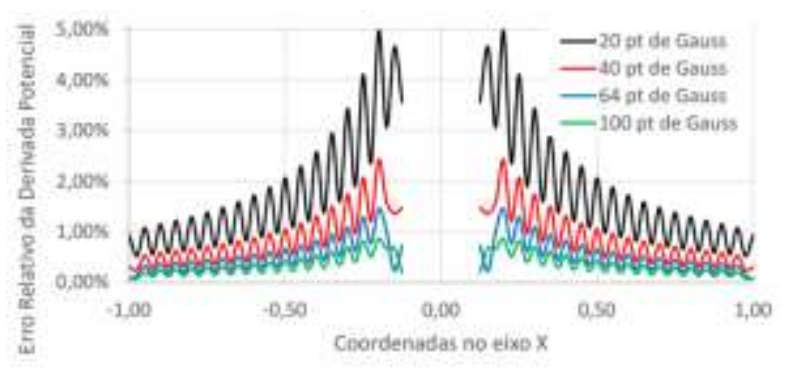

Figura 13-Erro Relativo da Derivada Potencial no exemplo 2.

\section{Referências}

[1] TANAKA, M.; SLADEK, V.; SLADEK V. Regularization Techniques Applied to Boundary Element Methods. Appl. Mech. Rev., v. 47, n. 10, p. 457-499, 1994.

[2] BREBBIA, C.A.; TELLES, J.C.F.; WROBEL, L.C. Boundary Element Techniques. New York: SpringerVerlag: 1984.

[3] PRESS, W. H.; TEUKOLSKY, S. A.; VETTERLING, W. T.; FLANNERY B. P. Numerical Recipes in Fortran 77, the Art of Scientific Computing, second Edition. New York: Cambridge University Press: 1996.

[4] BREBBIA, C.A.; WALKER, S. Boundary Element Techniques in Engineering. London: NewnesButterworths: 1980.

[5] KYTHE, P.K. An Introduction to boundary Element Methods. London: CRC Press: 1995.

[6] BANERJEE, P.K. The Boundary Element Methods in Engineering. London: McGraw Hill Book: 1994.

[7] BREBBIA, C. A.; DOMíNGUEZ J. The Boundary Element Method - An Introductory Course. UK: WIT Press: 1998.

[8] PESSOLANI, R. B. V.; MANSUR, W. J. Procedimento Adaptativo para Geometria definida por B-splines aplicado ao Método dos Elementos de Contorno. Revista Brasileira de Ciências Mecânicas, v. XIX, n.4, p. 504-517, 1997. 